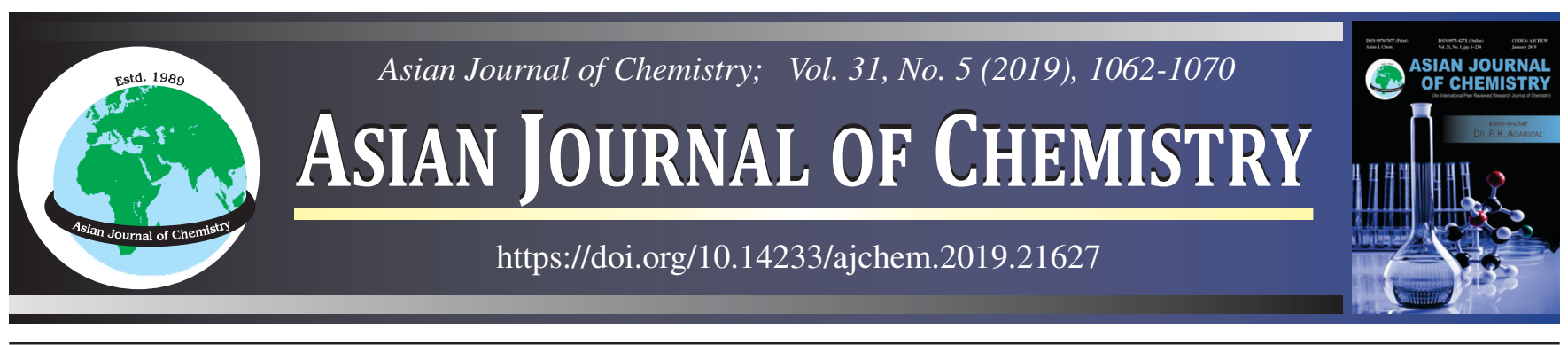

\title{
in situ Fabrication of Biological Chitosan and Gelatin-Based Hydrogels Loading Biphasic Calcium Phosphate Nanoparticles for Bone Tissue Regeneration
}

\author{
Tien Thinh Nguyen ${ }^{1,2}$, Chan Khon Huynh ${ }^{3}$, Van Thu Le ${ }^{2}$, Minh Dung Truong ${ }^{4}$, \\ Bach Long Giang ${ }^{5, *}$, Ngoc Quyen Tran ${ }^{2,4,5, * \bullet}$ and Minh Thanh Vu ${ }^{6, *}$
}

${ }^{1}$ Department of Pharmacy and Medicine, TraVinh University, TraVinh City 940000, Vietnam

${ }^{2}$ Graduate University of Science and Technology, Vietnam Academy of Science and Technology, Ho Chi Minh City 700000, Vietnam

${ }^{3}$ Biomedical Engineering Department, Ho Chi Minh City International University, Ho Chi Minh City 700000, Vietnam

${ }^{4}$ Institute of Applied Materials Science, Vietnam Academy of Science and Technology, Ho Chi Minh City 700000, Vietnam

${ }^{5}$ NTT Hi-Tech Institute, Nguyen Tat Thanh University, Ho Chi Minh City 700000, Vietnam

${ }^{6}$ Institute of Chemistry and Materials, 17 Hoang Sam, CauGiay, Hanoi 400000, Vietnam

*Corresponding authors: E-mail: tnquyen979@gmail.com; blgiang@ntt.edu.vn; vmthanh222@yahoo.com

Received: 1 August 2018;

Accepted: 28 December 2018;

Published online: 28 March 2019;

AJC-19331

| Adjustably biodegradable materials have gained much attention in biomedical applications. Among of them, various hydrogel-based scaffolds have applied for regenerating soft and hard tissues. In this study, according to differently biological properties of gelatin or chitosan as well as biphasic calcium phosphate nanoparticles (BCPNPs), several injectable nanocomposite hydrogels (INgel) were enzymatically fabricated from a phenolic chitosan derivative (PCD), phenolic gelatin derivative (PGD) and BCPNPs. According the change of $\mathrm{H}_{2} \mathrm{O}_{2}$ concentration with follow-up the time, the in situ formation of INgel was varied from 35 to $80 \mathrm{~s}$. The degradation rate of | the nanocomposite materials significantly related to in presence of collagenase that expended from 3 days to over one month depending on amount of the formulated PCD. The BCPNPs-encapsulated PCD-PGD INgel enhanced mineralization in the simulated biofluid. Fluorescent cytotoxicity assay indicated that the INgel was fabricated from a higher amount of the PGD resulting in a significant proliferation of bone marrow mesenchymal stem cells. These preliminary results exhibited a great potential of the INgel for bone regeneration.

| Keywords: Chitosan, Gelatin, Nanocomposite hydrogel, Collagenase, Bone regeneration.

ᄂ - - - - - - - - - - - - - - - - - - - - - - - - - -

\section{INTRODUCTION}

Recently, there has been a high demand of biomaterials in treatment or replacement of damaged tissues and organs leading to many studies on several advanced biocompatible and biodegradable materials [1]. Regarding the trend, hydrogel scaffolds play an important role in biomedical application due to their practical performance such as delivery of bioactive components and/or regenerative cells as well as encapsulation of nanoparticles in order to enhance cell attachment and proliferation $[2,3]$. The hydrogels consist of hydrophilic polymers that swell in aqueous solution, thus facilitating the transportation of substances such as nutrients and by-products from cell metabolism. Moreover, these materials could be welldesigned to be implanted in a minimally invasive surgical operation, improve patient compliance and degrade along with regeneration process of typical tissues [4-7]. Up to now, injectable hydrogel scaffolds have been synthesized based on physical or chemical methods. Physical hydrogels are formed by hydrophobic interaction, stereocomplex effect, electrostatic interaction, photochemical reaction, Michael-type reaction, Schiffbase reaction and enzyme-mediated crosslinking reactions [813]. Every obtained scaffold has exhibited some particular points on physical property, speed of matrix dissolution, compatibility, etc. Preparation of the injectable horseradish peroxidase (HRP) enzyme-mediated hydrogel has recently been emerging as an effective method because it is a highly specific reaction which avoids side reactions or production of toxic by-products to cells and living body [5,9,14]. Several kinds of the tyramine or $p$-hydroxyl phenyl acetic-conjugated

This is an open access journal, and articles are distributed under the terms of the Creative Commons Attribution-NonCommercial-ShareAlike 4.0 (CC BY-NC-SA 4.0) International License which allows readers to freely read, download, copy, distribute, print, search, or link to the full texts of its articles and to use them for any other lawful non-commercial purpose as long as the original source is duly acknowledged. 
polysaccharides (chitosan, dextran, hyaluronic acid, chitosan, heparin and chondroitin sulfate) are playing an important role in the injectable enzyme-mediated preparation of the highly biocompatible and biodegradable hydrogels that exhibited a great potential in tissue regeneration [5,15-20].

For hard tissue regeneration, these injectable hydrogel scaffolds have also performed much potential owing to their similarity to the extracellular matrix for osteoblast proliferation and migration. The injectable property completely fill up the defect site. However, bioactive properties of the hydrogels for bone regeneration are quite low. Combination of mineral nanoparticles and the hydrogels has recently been a new strend in fabricating nanocomposite hydrogels for hard tissue substitutes such as dentistry, orthopedics and reconstructive surgery. The nanocomposite hydrogels improved mechanical properties and some biological functions as compared with the original hydrogels [21]. In fact, annealed nano-hydroxyapatite-loaded polyvinyl alcol and poly acrylic acid (or polyvinylpyrolidone) composite hydrogel exhibited good thermostability, strength and mechanical properties $[22,23]$. Beta-tricalcium phosphatedispersed hydrogels significantly improved the compressive strength and biomineralization in simulated body fluid. Moreover, the vancomycin-encapsulated nanocomposite hydrogel performed antimicrobial activity against Staphylococcus aureus [24]. Photo-crosslinking bioactive glass-loaded polyethylene glycol dimethylmethacrylate hydrogels overcame an inherent brittleness of the bulky bioglass and enhanced biomineralization of the polymeric hydrogels [25]. In past, polysaccharide-based nanocomposite hydrogels have also paid much attention due to biocompatibility of the platform. Thermo responsive bioactive glass nanoparticles-reinforced chitosan and collagen hydrogel significantly increased stiffness of chitosan-based materials. The nanocomposite hydrogels were also compatible with human cells [26]. Chitosan-tetronictyramine copolymer used to disperse biphasic calcium phosphate (BCP) nanoparticles and prepare the HRP enzyme-mediated nanocomposite hydrogels exhibiting a great potential in biomedical applications. The composite hydrogels showed an increment in compressive strength and a high adhesion density of mesenchymal stem cells as compared to the hydrogel platform $[27,28]$. In general, these natural polymer-based nanocomposite hydrogels show good biocompatibility and mechanical properties. However, their biodegradation and support of cellular activities have not been focused [29].

It is well known that chitosan is tissue adhesive, hemostatic, anti-infective, biodegradable and supportive for cell attachment. However, not all cell types show good proliferation on chitosan based scaffolds [30-32]. Collagen and its denatured gelatin are widely used for pharmaceutical and medical applications due to their high biocompatibility, fast biodegradability and enhancement of cell attachment and proliferation $[33,34]$. Gelatin possesses more integrin binding domains for cell attachment and thus enhances cell attachment. Nonetheless, they are quickly degraded by collagenase within 3-4 days [34-36]. Calcium phosphate nanoparticles have been used in orthopedic applications because of its biocompatibility, osteoconductivity and osteointegration [37]. Biphasic calcium phosphate has been reported as more efficient in repairing periodontal defects and producing osteo induction as compared to hydroxyapatite or tricalcium phosphate. The BCP nanoparticles can also perform an appropriate biodegradation and osteointegration [38]. However, brittleness of the BCP materials partially reduced its potential in bone applications [39]. Therefore, fabrication of the polymeric $\mathrm{BCP}$ nanocomposite biomaterials has been an approach $[39,40]$.

In this study, an in situ-forming nanocomposite hydrogels prepared from 4-hydroxyphenylacetamide-conjugated chitosan and tyramine-functionalized gelatin in the presence of the BCPNPs, HRP enzyme and small amount of $\mathrm{H}_{2} \mathrm{O}_{2}$. The chitosan/ gelatin and BCPNPs-based INgels could be adjustable gelation time, appropriate collagenase-mediated degradation rates and enhancement of biomineralization and bone cell growth that enable it to be a great plaform for regenerative medicine to overcome some limitations of gelatin or chitosan-based materials.

\section{EXPERIMENTAL}

Chitosan low molecular weight (75-85\% deacetylation), gelatin type A from porcine skin (Bloom 300), 4-hydroxylphenylacetic acid (HPA) and tyramine (TA), 1-ethyl-3-(3dimethylaminopropyl) carbodiimide (EDC) were obtained from Acros Organics. Collagenase and horseradish peroxidase (HRP) enzymes (type VI, 298 purpurogallin unit/mg solid) were purchased from Sigma-Aldrich.

\section{Preparation of polymers and BCPNPs}

Preparation of PCD and PGD: PCD and PGD derivatives were prepared from chitosan and gelatin using carbodiimide coupling reagent. Briefly, chitosan $(1 \mathrm{~g})$ was dissolved in a solution of $40 \mathrm{~mL}$ distilled (DI) water and $0.5 \mathrm{~mL} \mathrm{HCl} 1.0$ M. HPA ( $0.45 \mathrm{~g}, 2.9 \mathrm{mmol})$ was added into the mixture. $\mathrm{pH}$ of solution was adjusted to 5 and then EDC $(0.90 \mathrm{~g}, 4.7 \mathrm{mmol})$ was added to the chitosan solution under stirring for $24 \mathrm{~h}$ at room temperature. The solution was dialyzed against DI water using membrane dialysis (molecular weight Cut-Off (MWCO) 6000-8000) for 3 days. Subsequently, the modified chitosan solution was lyophilized to obtain PCD as demonstrated in Fig. 1a. The obtained PGD was $0.94 \mathrm{~g}$ and content of HPA was $0.068 \mathrm{wt} / \mathrm{wt} \%$ (calculated by UV-vis). ${ }^{1} \mathrm{H}$ NMR $\left(\mathrm{D}_{2} \mathrm{O}\right) / \mathrm{ppm}$ : $\delta 2.05$ (s, $-\mathrm{COCH}_{3}$, chitosan); $\delta 3.22\left(\mathrm{~m},-\mathrm{C}_{2}(\mathrm{H})\right.$, chitosan); $\delta$ 3.43-3.92 ( $\mathrm{m}, \mathrm{C}_{3+4+5+6}$, chitosan); $\delta 2.89\left(\mathrm{~d},-\mathrm{CH}_{2^{-}}, \mathrm{HPA}\right) ; \delta 6.89$ and $7.22(\mathrm{~d},-\mathrm{CH}=\mathrm{CH}-, \mathrm{HPA})$ [41].

Gelatin $(2 \mathrm{~g})$ and tyramine $(1.00 \mathrm{~g}, 7.3 \mathrm{mmol})$ were dissolved in distilled water $(30 \mathrm{~mL})$. The $\mathrm{pH}$ of the mixture was adjusted to 6 following addition of EDC $(0.50 \mathrm{~g}, 2.5 \mathrm{mmol})$ under stirring for $24 \mathrm{~h}$. Then, the solution was dialyzed against deionized water using membrane dialysis (MWCO 6000-8000) for 3 days. Subsequently, the dialyzed solution was freeze-dried to obtain PGD as demonstrated in Fig. 1b. The obtained PGD was $1.80 \mathrm{~g}$ and content of tyramine was $0.01 \mathrm{wt} / \mathrm{wt} \%$ (calculated by UV-vis). ${ }^{1} \mathrm{H} \mathrm{NMR}\left(\mathrm{D}_{2} \mathrm{O}\right) / \mathrm{ppm}$ : $\delta 6.75$ and $7.11(\mathrm{~d},-\mathrm{CH}=\mathrm{CH}-$ of tyramine), $\delta 2.65$ and 2.88 (m, $-\mathrm{CH}_{2} \mathrm{CH}_{2}-$, tyramine).

Preparation of BCP nanoparticles: Recent years, BCPNPs have been widely studied for bone regeneration due to its biocompatibility and osteoconductivity. In our previous study, the nanoparticles were synthesized using calcium chloride and tricalcium phosphate salts at molar ratio of $\mathrm{Ca} / \mathrm{P}=1.57$. The 


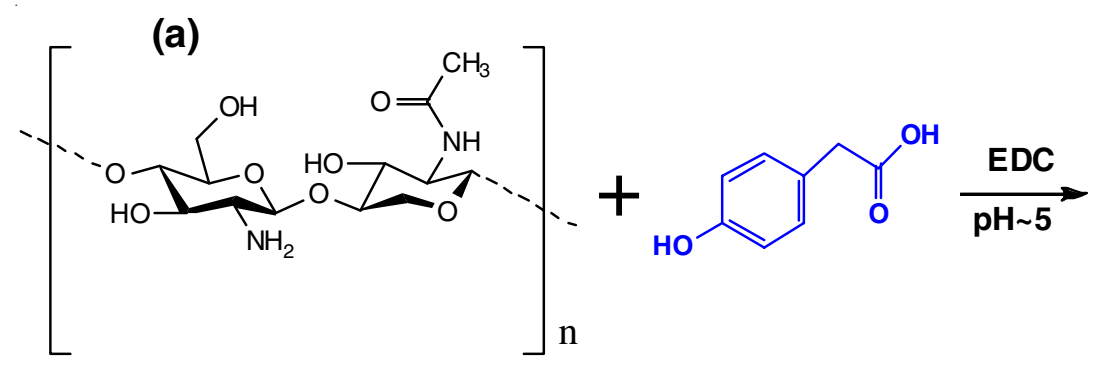

Chitosan
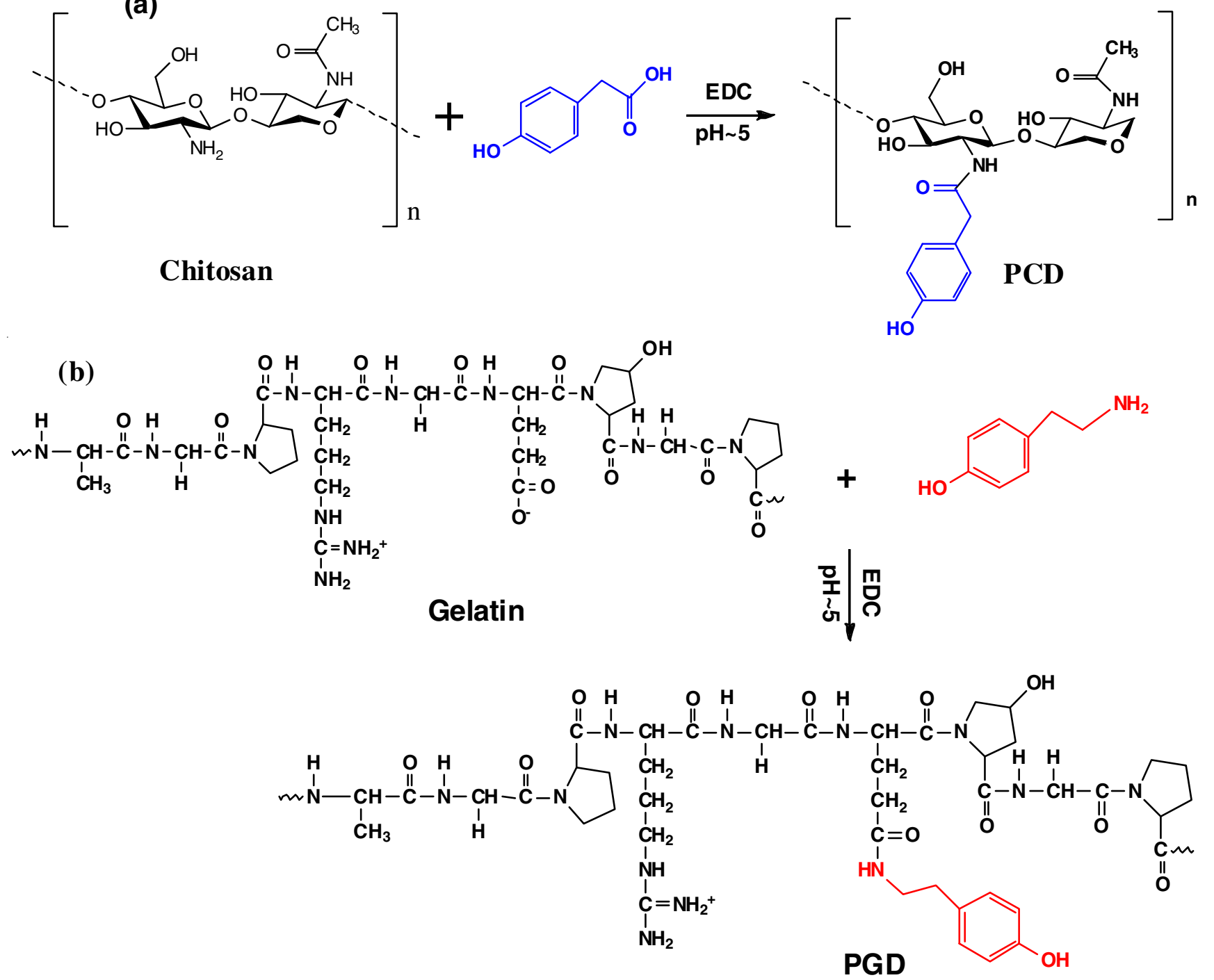

Fig. 1. Synthetic scheme of PCD (a) and PGD (b)

$\mathrm{pH}$ of the reaction mixture was maintained at $\mathrm{pH}$ 7. Calcination process was conducted at $750{ }^{\circ} \mathrm{C}$ to obtain $\mathrm{BCP}$ material. The BCPNPs obtained below $80 \mathrm{~nm}$ in diameter via ball-milling process [27].

Preparation of PGD-PCD-based hydrogels and INgels: Preparation of gelatin or chitosan-based hydrogels was evaluated as some screening experiments to select a suitable condition for further works. Briefly, PGD $(40 \mathrm{mg})$ was dissolved in distilled water $(300 \mu \mathrm{L})$ and separated into two vials equally. Then, enzyme HRP ( $30 \mu \mathrm{L}$ of $0.05 \mathrm{mg} / \mathrm{mL}$ stock solution) and $\mathrm{H}_{2} \mathrm{O}_{2}$ (30 $\mu \mathrm{L}$ of $0.05-0.15 \% \mathrm{w} / \mathrm{v}$ stock solution) were added into each tube separately. The gelatin-based hydrogel was formed by mixing two $\mathrm{HRP}$ and $\mathrm{H}_{2} \mathrm{O}_{2}$-contained vials. $\mathrm{PGD}$ polymer concentration was $10 \% \mathrm{w} / \mathrm{w}$ in hydrogel. Chitosan-based hydrogel was prepared by the same process as described for gelatin-based hydrogel in which $8 \mathrm{mg}$ PCD was prepared in distilled water $(150 \mu \mathrm{L})$ with $\mathrm{HRP}(30 \mu \mathrm{L}$ of $0.05 \mathrm{mg} / \mathrm{mL}$ stock solution) and $\mathrm{H}_{2} \mathrm{O}_{2}(30 \mu \mathrm{L}$ of $0.05-0.15 \%$ w/v stock solution). The final concentration of the polymers solution was $8 \% \mathrm{w} / \mathrm{w}$. The gelation time was determined by using the vial tilting method.

For PGD-PCD hydrogels, in situ hydrogel formation occurred when solution A (contained PGD, PCD and HRP) mixed with solution B (contained PGD, PCD and $\mathrm{H}_{2} \mathrm{O}_{2}$ ) at same volume of the precursor polymer solution as demonstrated in the above experiments.

BCPNPs-dispersed PGD-PCD hydrogels were prepared via the same process of the hydrogels, in which contained 10 w/w \% of the BCPNPs. The gelation time behaviours of the hydrogels and INgels were characterized at the different concentration of $\mathrm{H}_{2} \mathrm{O}_{2}$ from 0.05 to $0.15 \% \mathrm{wt} / \mathrm{vol}$. Morphology of the INgel was observed by scanning electron microscopy (SEM) at the freeze-dried form. Depending on weighted amount of the conjugated tyramine and HPA moieties in the modified polymers, concentrations of $\mathrm{HRP}$ and $\mathrm{H}_{2} \mathrm{O}_{2}$ were adjusted to prepare the hydrogels or nanocomposite hydrogels. The enzymemediated hydrogels contained approximately $8 \mathrm{w} / \mathrm{w} \%$ of the polymer concentration. Effect of $\mathrm{PCD}$-formulated weight ratio on the collagenase-mediated biodegradation of biomaterials were investigated.

Collagenase-mediated degradation study: The biodegradation of hydrogels and INgels was studied in a collagenase media, in which these materials were immersed in phosphate buffer saline (PBS) solution $\mathrm{pH} 7.4$ containing collagenase $(0.2 \mathrm{U} / \mathrm{mL})$ at $37^{\circ} \mathrm{C}$ and then monitored their weight-losses at 
different incubation times. Samples with different mass ratios were accurately weighted $\left(\mathrm{w}_{\mathrm{i}}\right)$ before immersing in $1 \mathrm{~mL}$ of enzymatic solution. At the predetermined intervals, samples were removed from the incubation medium. Then, weight of remaining hydrogels and INgels $\left(\mathrm{w}_{\mathrm{t}}\right)$ were measured.

$$
\text { Degradation rate (rate of weight loss } \%)=\frac{\mathrm{W}_{\mathrm{i}}-\mathrm{W}_{\mathrm{t}}}{\mathrm{W}_{\mathrm{i}}} \times 100
$$

where $\mathrm{W}_{\mathrm{i}}$ and $\mathrm{W}_{\mathrm{t}}$ are initial or remaining weights of hydrogels or INgels, respectively).

Biomineralization study: Biomineralization is one of the most crucial factors to evaluate potential of materials for bone regeneration. In the study, the PGD-PCD hydrogels and its nanocomposites were immersed in a SBF buffer solution $(\mathrm{pH}$ 7.4). After 4 weeks soaking in the SBF solution, these materials were collected and washed with distilled water to remove soluble inorganic salts. Biomineralization of the INgels were characterized by scanning elecgron microscopy, energy dispersive X-ray analysis and X-ray diffraction methods.

Evaluation of cytocompatibility: The rabbit bone marrow (MSCs) were cultured in $\alpha$-minimum essential medium $(\alpha-$ MEM) supplemented with $10 \%$ fetal bovine serum, $100 \mathrm{U} / \mathrm{mL}$ penicillin $\mathrm{G}$ and $100 \mu \mathrm{g} / \mathrm{mL}$ streptomycin (Gibco BRL, NY, USA). The MSCs were seeded at 10 million cells per $\varnothing 150$ mm dish and cultured at $37^{\circ} \mathrm{C}$ in a $5 \% \mathrm{CO}_{2}$ incubator. The culture medium was replaced every three days. Once cells reached 80 $\%$ confluence, they were detached by using Trypsin-EDTA (Gibco BRL, NY, USA) and re-suspended in $\alpha$-MEM.

Cytocompatible study was conducted to evaluate growth of mesenchymal stem cells derived from rabbit bone marrow (MSCs) in the INgels. $5 \times 10^{4} \mathrm{MSC}$ cells were seeded onto the UV-sterilized samples in 24-well plates. After $48 \mathrm{~h}$ incubation, these cellular materials were washed with PBS three times. Cell viability was evaluated by commercially available live/ dead viability/cytotoxicity kit (Life Technologies Korea LLC, Seoul, Korea). The results were observed using fluorescence microscope (TE2000, Nikon, Seoul, Korea) equipped with a digital camera.

Characterizations: The structure of PCD and PGD were determined by using NMR spectrometer (Varian, $400 \mathrm{MHz}$, U.S.A). Contents of HPA and tyramine in the natural polymers were evaluated by UV-vis measurement (spectrophotometer JASCO V-570, Japan). Morphology of the BCPNPs and INgels were observed by Field-emission scanning electron microscope (FESEM, JSM-635F, JEOL). The phase analysis of the mineralization layer was identified using an X-ray diffractometer, D8/Advance, Bruker, UK) with $\mathrm{CuK}_{\alpha},(\lambda=1.5406 \AA)$ as a radiation source over the $2 \theta$ range of $10-70^{\circ}$ at $25^{\circ} \mathrm{C}$ ). Compositions of the phase were analyzed by energy dispersive X-ray (EDX) analysis. The measurement was tested on the carboncoated samples.

\section{RESULTS AND DISCUSSION}

Characterizations of polymers: The HPR-mediated crosslinking reactions play a crucial role in preparation of several natural polymers-based hydrogels $[5,8,9]$. PGD was synthesized by the coupling reaction using EDC. The gelatin derivative was determined by the resonance signals ( 2.65 and $2.88 \mathrm{ppm}$ ) of the methylene protons of tyramine. Peaks of aromatic protons of tyramine appeared at 6.75 and $7.11 \mathrm{ppm}$. Some signals of amino acids in ${ }^{1} \mathrm{H}$ NMR spectrum were shown (Fig. 2a): $\delta 4.55$ and 4.68 (- $\mathrm{CH}_{2^{-}}$, proline); 4.27 (-CH-, hydroxyproline); $3.88\left(-\mathrm{CH}_{2-}\right.$, alanine $) ; 1.34\left(-\mathrm{CH}_{3}\right.$, alanine); 3.57 (- $\mathrm{CH}_{2-}$, glycine); 2.23 (- $\mathrm{CH}_{2^{-}}$, glutamic acid); $1.60\left(-\mathrm{CH}_{2^{-}}\right.$, arginine); 3.14, 7.23 and 7.29 (-CH-, phenylalanine).

$\mathrm{HPa}$ conjugated with chitosan formed its soluble PCD for in situ preparation of the hydrogels. The PCD was confirmed from resonance signals of aromatic protons of $\mathrm{HPa}$ at 6.89 and $7.22 \mathrm{ppm}$ (Fig. 2b). The signals at $2.89 \mathrm{ppm}$ were assigned to methylene protons of HPa. Overlapped and broad resonance signals of D-glucosamine of chitosan were observed in the interval 3-4 ppm. These ${ }^{1} \mathrm{H}$ NMR results could confirm the successful preparation of two phenolic precursor polymers for fabricating the HRP enzyme-mediated hydrogels.

Characterizations of BCP: Recent years, BCPNPs have considered as one of most important calcium phosphate materials in bone reconstruction. There are two common methods for preparing the nanoparticles such as sintering of a calcium phosphate salt precipitate state from aqueous reaction and solid-state reactions between two inorganic calcium and phosphate salts. In the study, the BCP nano powders were synthesized using ultrasound irradiation assisted process prior to the sintering. The synthesized BCP powders had a higher uniformity and spherical shape with diameter around $80 \mathrm{~nm}$ as seen in Fig. 3a. X-rat diffraction showed two crystalline phases
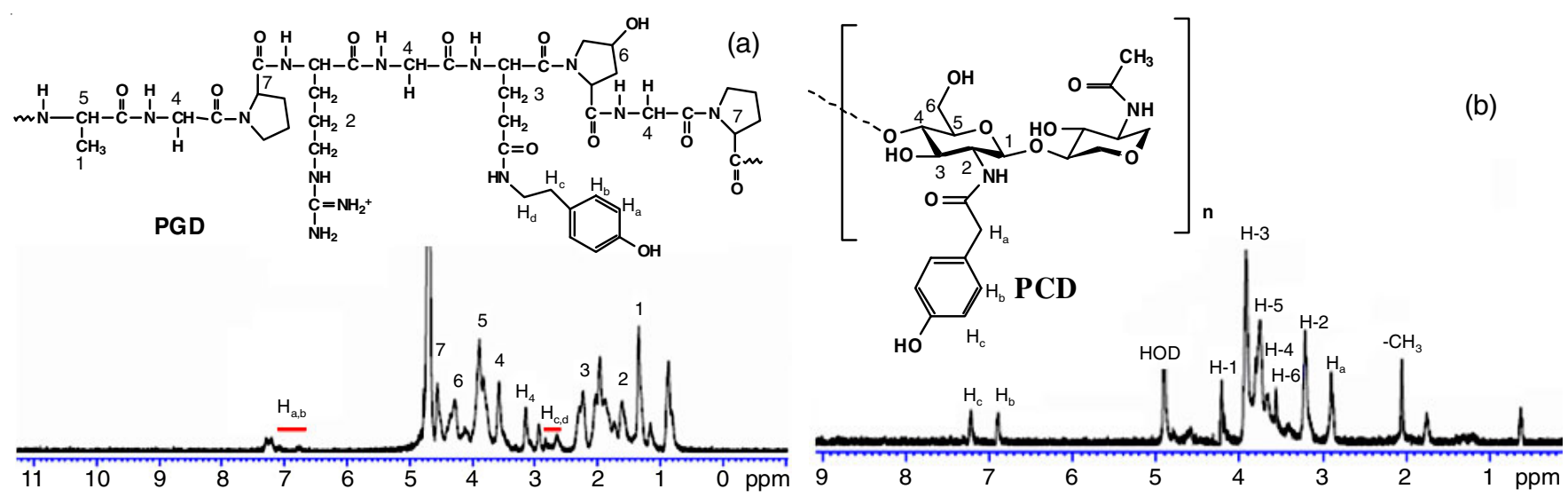

Fig. 2. ${ }^{1} \mathrm{H}$ NMR spectrum of PGD (a) and PCD (b) in $\mathrm{D}_{2} \mathrm{O}$ 

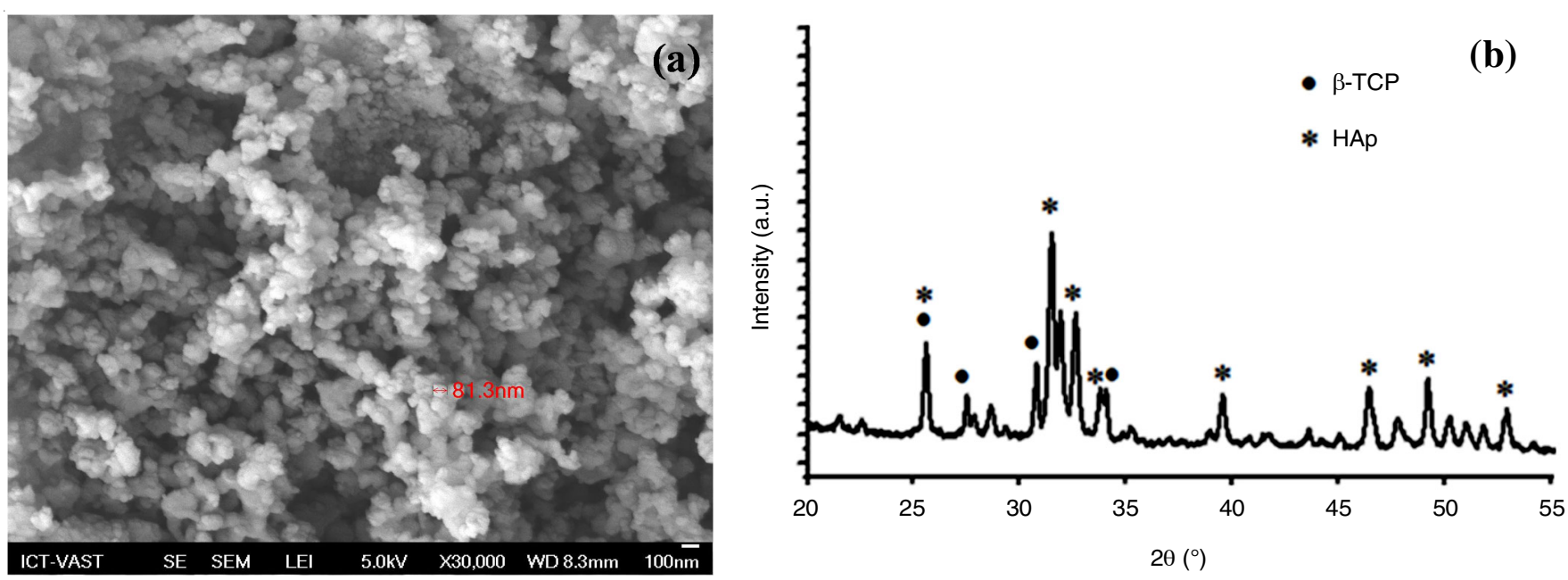

Fig. 3. Morphology (left) and phase analysis of BCPNPs (right) by SEM image and X-ray diffraction, respectively

of $\beta$-TCP and HAp (Fig. 3b). With the small particle size, the nanoparticles will be highly potential in fabrication of nanocomposite biomaterials.

Characterizations of hydrogels, INgels and gelation time: Regarding to several previous studies, $\mathrm{HRP} / \mathrm{H}_{2} \mathrm{O}_{2}$-mediated coupling reaction of phenolic moities-modified polymers is an high efficieny to prepare in situ injectable hydrogel [5]. For preparation of the hydrogels in screening conditions, the fast gelation time obtained to be $60 \mathrm{~s}$ and $12 \mathrm{~s}$ for PGD and PCD solution at around $0.125 \mathrm{wt} \%$ of the stock $\mathrm{H}_{2} \mathrm{O}_{2}$ concentration (or $0.0625 \mathrm{wt} \%$ in a final concentration), respectively. Amount of the $\mathrm{H}_{2} \mathrm{O}_{2}$ catalyst in the preparation was used in regard to the UV-vis-determined HPA or tyramine content. It was reported that to obtain the fast gelation time, a molar ratio of $\mathrm{H}_{2} \mathrm{O}_{2}$ and phenolic moiety at 0.5 is optimal $[5,9,11,16]$. Regarding these reports, HRP catalyzes to decompose hydrogen peroxide for coupling phenol or aniline derivatives. The minimally fed HRP performed effectively at very low content (about $0.005 \mathrm{mg} / \mathrm{mL}$ of the enzyme in final concentration). Understanding the role of HRP enzyme is very critical for formulation and fabrication of several types of phenolic derivatives-based biomaterials. Regarding the obtained results, the CTA and PCD-based hydrogels and INgels were prepared as demonstrated in Fig. 4a. $\mathrm{H}_{2} \mathrm{O}_{2}$ concentration significantly affected the gelation time of PCD-PGD (1:5 wt/wt) hydrogel and its nanocomposite as seen in Fig. 4b. In the investigated concentration range of the stock $\mathrm{H}_{2} \mathrm{O}_{2}$, an increment of the catalyst content induced a fast gelation of the polymers solution in presence of HRP that could be explained by a higher content of the produced hydroxy radical for oxidizing phenolic moieties to crosslink polymer chains. Previous studies have shown that an excess amount of $\mathrm{H}_{2} \mathrm{O}_{2}$ could be harmful to catalytic role of HRP and induce cytotoxicity $[5,11,31]$. The gelation time of the polymer solutions seemed to be constant at 0.125 and $0.15 \mathrm{wt} \%$ of the used $\mathrm{H}_{2} \mathrm{O}_{2}$ concentration so the preparation of PCD-PGD (1:5 w/w)based materials should select at $0.125 \mathrm{wt} \%$ of $\mathrm{H}_{2} \mathrm{O}_{2}$ concentration as an optimal condition. The selected $\mathrm{H}_{2} \mathrm{O}_{2}$ concentration was enough to oxidize phenolic moiety and significantly decreased in the crosslinking process that couldn't induce cell apoptosis. In general, PCD/PGD hydrogels and INgels could be formed quickly in situ within $40 \mathrm{~s}$. However, there is a slightly difference in gelation time of the hydrogels and theirs nanocomposites that contain BCPNPs. That is due to free functional groups of gelatin and chitosan binding to $\mathrm{OH}$ groups in BCP resulting in increasing crosslinking density of INgels and thus decreased its gelation time. A further study on the INgels's porosity was conducted. Fig. $4 \mathrm{c}$ shows pore-size distribution of the material ranging from 100 to $500 \mu \mathrm{m}$. BCPNPs were well-distributed in the scaffold as seen in Fig. 4d. These results indicate supportive effects on cell growth and migration or sustainable release of bioactive components of the INgels [9,42].

in vitro Biodegradation study: Biodegradability of several artificial materials plays an important role in the tissue regeneration or drug delivery systems. For implanted materials, studies on their enzymes-induced biodegradation are especially important to select a degradable scaffold with repairing or regenerating process of the defects. As mentioned, biodegradation and cellular compatibility behaviours of the chitosan or gelatinbased biomaterials are partially different. Fig. 5a shows the collagenase-induced degradation profiles that differently performed as changed mass ratios of PCD-PGD in the gels. The degradation rate decreased following a decrement in amount of gelatin in the PCD-PGD-based hydrogels. The hydrogels at a mass ratio of 0 PCD: 10 PGD were completely degraded after $42 \mathrm{~h}$ whereas at ratio of 0.5 PCD: 10 PGD, a complete degradation took $90 \mathrm{~h}$. In contrast, the higher chitosan-formulated hydrogels (1 PCD: 10 PGD; 1 PCD: 5 PGD; 1 PCD: 2.5 PGD) were not utterly degraded within $762 \mathrm{~h}$. The collagenase-induced degradation of the INgels was partially slower than that of the hydrogels as compared from 0.5 PCD: 10 PGD samples (Fig. 5b). The INgel prepared at 1 PCD: 5 PGD and 1 PCD: 2.5 PGD ratios performed a lowest degradation rate approximately $60 \mathrm{wt} / \mathrm{wt} \%$ of its weight at the end of this survey. The different behaviours could be derived from binding of calcium ions (released from BCPNPs) and collagenase leading to inhibition of its proteolytic activity [43]. These studies clarified that gelatin-based materials have a fast biodegradable characteristic in a collagenase containing media while the biodegradation of chitosan-formulated hydrogel could be modulated. The obtained results are also significant because the hydrogels or its nanocomposite could be used to implant for regenerating every specific tissue. 

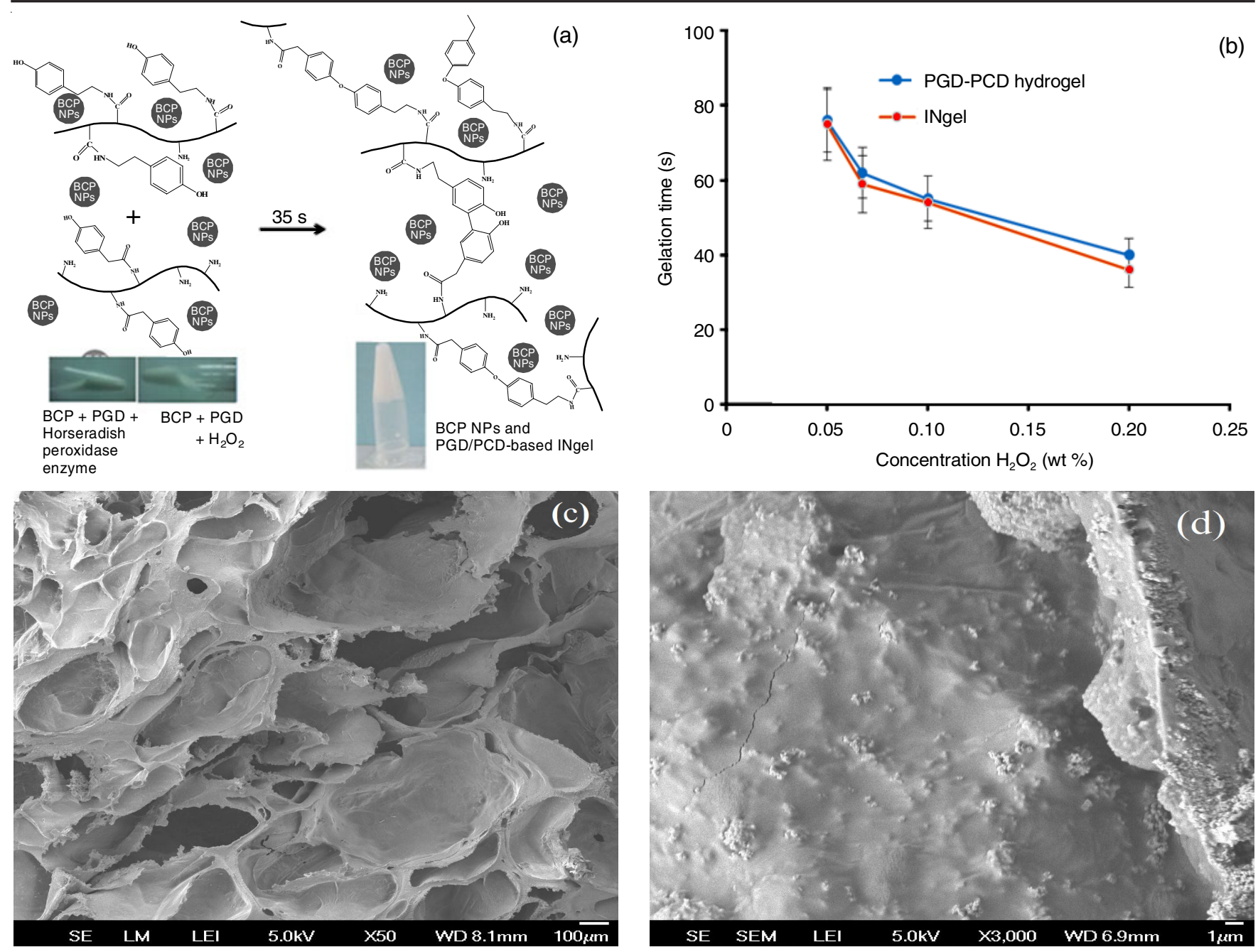

Fig. 4. Demonstration of in situ formation of the INgel in the presence of $0.05 \mathrm{mg} / \mathrm{mL} \mathrm{HRP}$ and 0.125 wt $\% \mathrm{H}_{2} \mathrm{O}_{2}$ (a); gelation time of the PGD-PCD (nanocomposite) hydrogels (b); morphologies of the freeze-dried INgels at different magnifications: x 50 (c) and x 3000 (d)

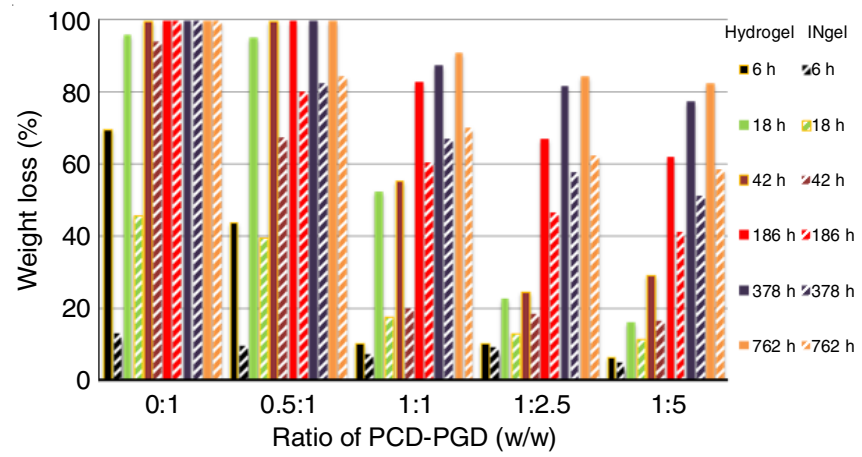

Fig. 5. Collagenase-induced biodegradation of the PCD-PGD hydrogels (a) and its INgels (b)

Biomineralization study: Biomineralization is one of the most crucial factors to evaluate potential of materials for bone regeneration. In the study, the PGD-PCD (5:1) and PGD-PCD (2.5:1) hydrogels and their nanocomposites were characterized due to their similar slow degradation rate and different amount of PGD. The studies could indicate whether formation of biomineralization is dependent on nature of polymers that forming hydrogel. Fig. 6a-b [profile (1) and (2)] show all initial hydrogels and these SBF-incubated samples are in amorphous phase without any diffraction peaks responding to $\mathrm{BCP}$ crystalline. XRD diffraction of the initial INgels showed several typical peaks presenting HAp and $\beta$-TCP crystallized phases in BCPNPs as seen in profiles (3). After 28 days of incubation, a highly crystallized phase of HAp could be observed in profile (4) and some diffraction peaks of calcium carbonate appeared which indicated a substitution of carbonate into HAp lattice that occurred in the SBF-mediated mineralization process leading formation of carbonated HAp on the surface of the incubated materials [44]. These results confirmed efficiency of the INgelss in enhancing biomineralization in vitro.

EDX analysis results in Fig. 7 indicated the material with a different PGD content exhibiting a distinct mineralization behaviour. The PGD-PCD $(5: 1)$ hydrogel formulated with a higher amount of PGD performed an increment in deposition of calcium and phosphate ions on its surface as compared to the PGD-PCD (2.5:1) hydrogel as seen in Fig. 7a and 7b. There was a same behaviour in two INgels (Fig. 7c and 7d). The PGDPCD (5:1)-formulated scaffold indicated a higher deposition of calcium and phosphate ions. Our data is aligned with previous report in which HAp-encapsulated gelatin matrix was shown to induce a significant formation of the carbonated HAp on scaffold surface after incubated in SBF solution for 21 days 

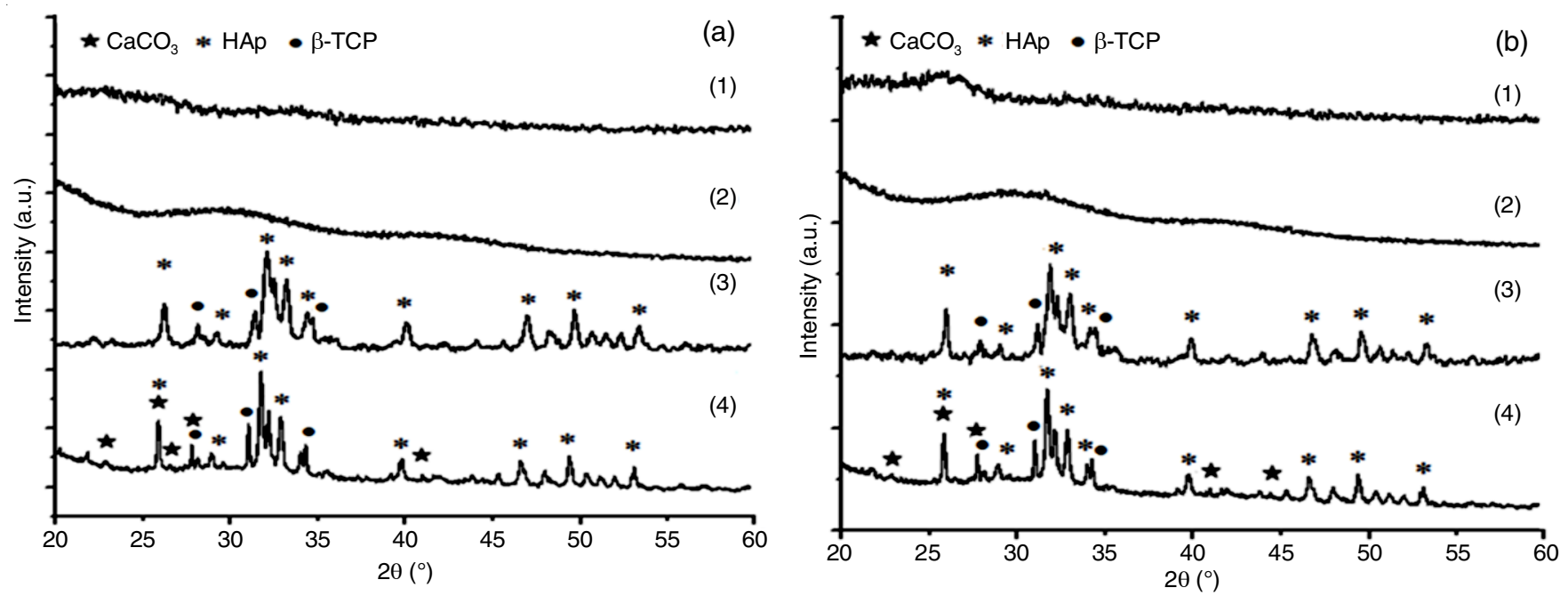

Fig. 6. XRD diffractograms of hydrogels (PGD-PCD (5:1; a), PGD-PCD (2.5:1; b)) and its INgels: Initial hydrogel (1), 28 days incubated hydrogel (2), initial INgels (3) and its 28 days incubated nanocom-posite sample (4)
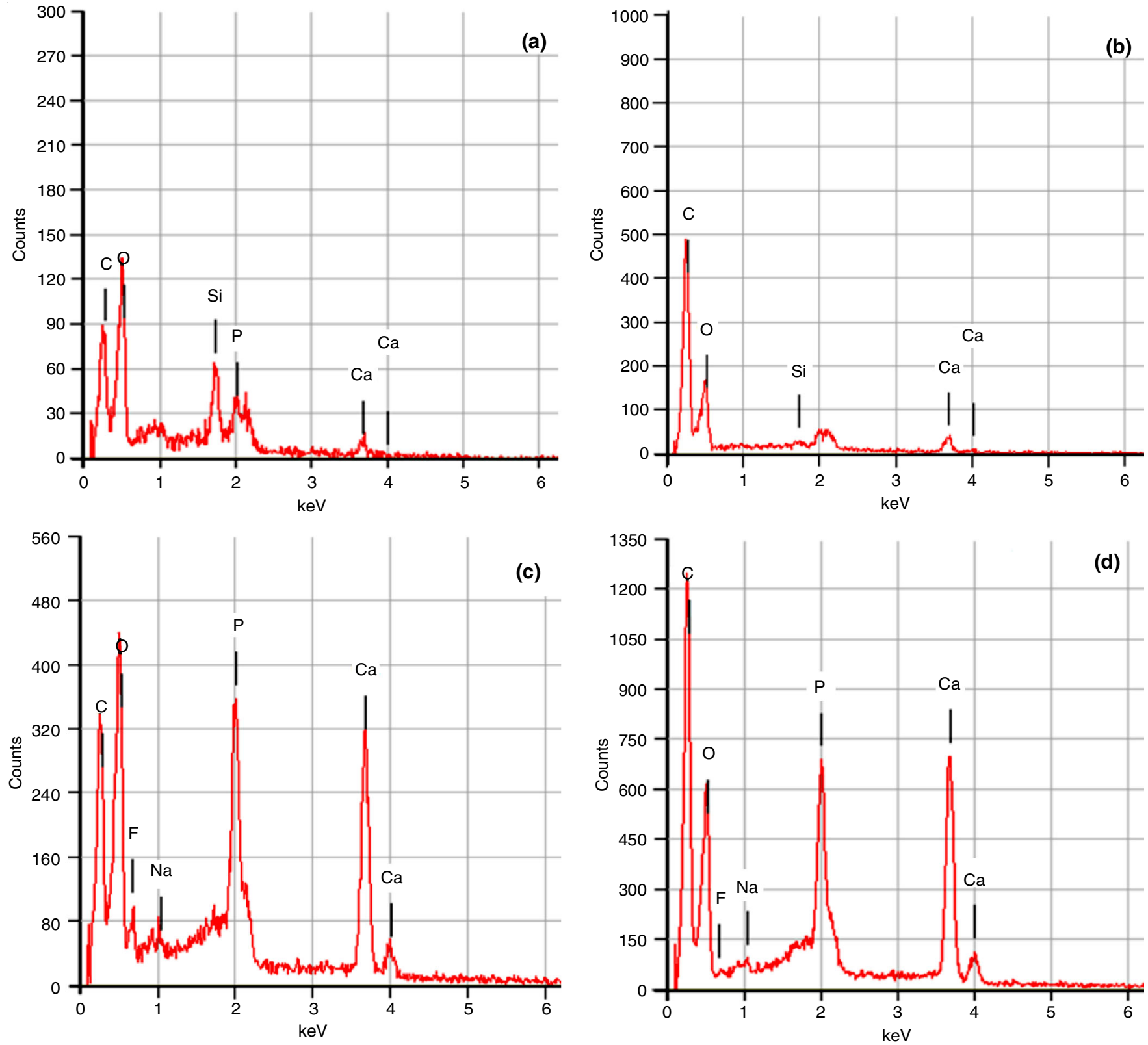

Fig. 7. EDX spectra of the hydrogels (PGD-PCD (5:1; a), PGD-PCD (2.5:1; b)) and its INgels after soaking 28 days 

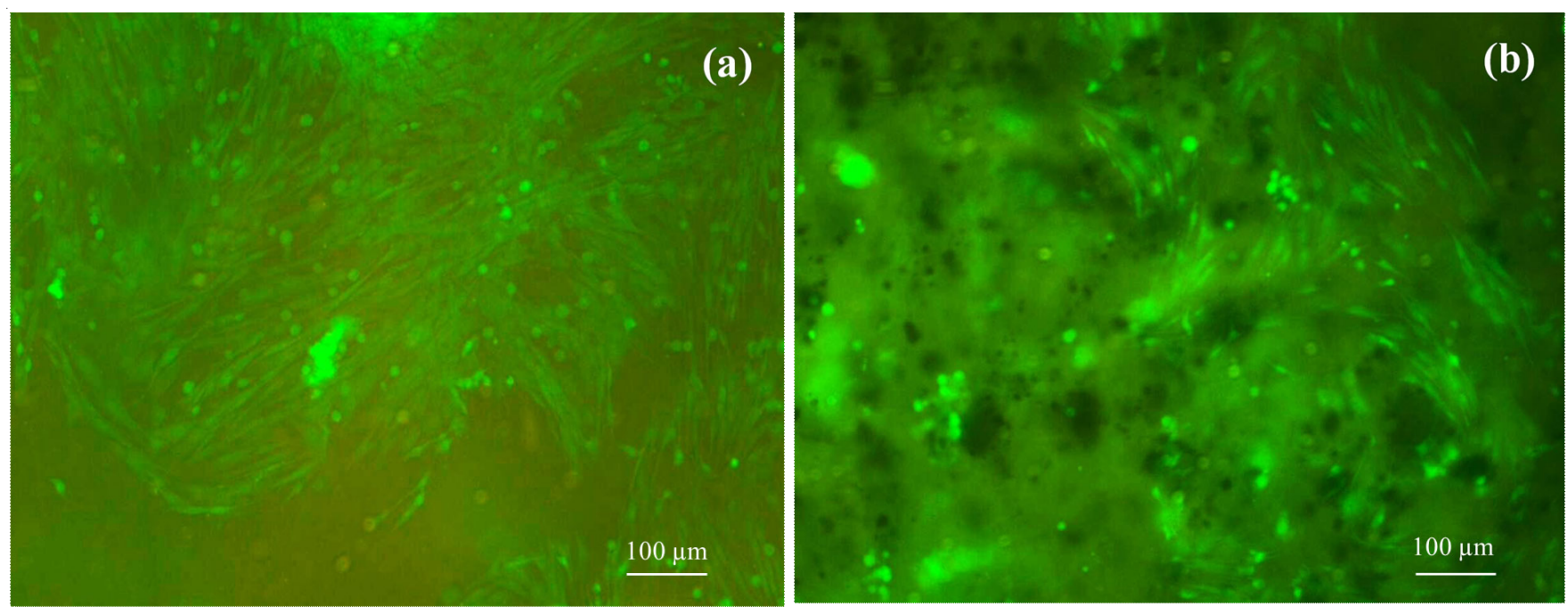

Fig. 8. Fluorescent microscopy images of the cellular PGD-PCD (5:1) INgels (a) and PGD-PCD (2.5:1) nanocomposite sample (b)

[45]. The obtained results confirm that the highly PGD or gelatin-formulated sample could enhance mineralization ability.

Cytocompatibility of INgels: In cytocompatible evaluation of hydogel scaffolds, live/dead staining assay is one of the most popular technique for visualizing behaviour of cells on the scaffolds. The living cells are stained with green fluorescence by an intracellular esterase reduction of a nonfluorescent calcein AM [9,31]. Fig. 8a and 8b show that the INgels were cytocompatible with Mesenchymal stem cells. This is similar to our previous study indicated that chitosan-tetronic/ BCP nanocomposite hydrogels could enhance MSCs attachment and a supplement of BCPNPs also contributed to accelerate proliferation of the cell due to its rough surface and a high protein deposition from the incubated media [27]. In the evaluation, a high density of the proliferated MSCs could observe on the PGD-PCD (5:1) INgel as compared to PGDPCD (2.5:1) nanocomposite sample. It is well-known that gelatin-enrich biomaterials could induce outgrowth of cells because gelatin owns more integrin binding domains for cell attachment and enhancing outgrowth of cells $[34,35]$. These results could offer several different formulations of the PGDPCD hydrogel with a various collagenase-mediated degradation rate and the high cytocompatibility which enables implant with minimally invasive ways exhibiting its greatly potential for regenerating several kinds of typical tissues as combined with bioactive molecules or/and nanoparticles such as growth factor, genes, BCPNPs, bioglass nanoparticles, etc.

\section{Conclusion}

In the study, the PCD-PGD/BCP INgels were in situ prepared via horseradish peroxidase-catalyzed reaction. Depending on the amount of formulated chitosan derivative, the INgels performed an appropriated biodegradation rate over a long period of time. Moreover, an increment of the formulated gelatin, the biocomposite could enhance biomineralization and MSCs proliferation on the composite surface. Regarding to these obtained results and some well-known osteo induction and osteo integration characteristics of BCPNPs, the study could pave a way for further evaluations to apply in bone tissue regeneration.

\section{ACKNOWLEDGEMENTS}

This work was financially supported by Tra Vinh University and VAST.

\section{CONFLICT OF INTEREST}

The authors declare that there is no conflict of interests regarding the publication of this article.

\section{REFERENCES}

1. Y. Yuan, D. Lin, F. Chen and C. Liu, J. Orthopaedic Transl., 2, 49 (2014); https://doi.org/10.1016/j.jot.2013.12.002.

2. Y. Li, J. Rodrigues and H. Tomás, Chem. Soc. Rev., 41, 2193 (2012); https://doi.org/10.1039/C1CS15203C.

3. R. Scaffaro, F. Lopresti, A. Maio, F. Sutera and L. Botta, J. Appl. Biomater. Funct. Mater., 15, 107 (2017); https://doi.org/10.5301/jabfm.5000332.

4. D. Schmaljohann, Adv. Drug Deliv. Rev., 58, 1655 (2006); https://doi.org/10.1016/j.addr.2006.09.020.

5. M. Kurisawa, J.E. Chung, Y.Y. Yang, S.J. Gao and H. Uyama, Chem. Commun., 34, 4312 (2005);

https://doi.org/10.1039/b506989k

6. T.B.T. Nguyen, L.H. Dang, T.T.T. Nguyen, D.L. Tran, D.H. Nguyen, V.T. Nguyen, C.K. Nguyen, T.H. Nguyen, V.T. Le and N.Q. Tran, Green Process. Synth., 5, 511 (2016); https://doi.org/10.1515/gps-2016-0062.

7. Y.L. Wu, H. Wang, Y.K. Qiu, S.S. Liow, Z. Li and X.J. Loh, Adv. Healthc. Mater, 5, 2679 (2016);

https://doi.org/10.1002/adhm.201600723.

8. N.Q. Tran, Y.K. Joung, E. Lih, K.M. Park and K.D. Park, Macromol. Res., 19, 300 (2011); https://doi.org/10.1007/s13233-011-0309-y.

9. D.H. Nguyen, N.Q. Tran and C.K. Nguyen, J. Biomater. Sci., 24, 1636 (2013); https://doi.org/10.1080/09205063.2013.789356.

10. J.H. Choi, Y.K. Joung, J.W. Bae, J.W. Choi, T.N. Quyen and K.D. Park, Macromol. Res., 19, 180 (2011); https://doi.org/10.1007/s13233-011-0214-4.

11. N.T. Phuong, V. Anh Ho, D. Hai Nguyen, N.C. Khoa, T.N. Quyen, Y. Lee and K.D. Park, J. Bioact. Compat. Polym., 30, 412 (2015); https://doi.org/10.1177/0883911515578760.

12. X.Z. Shu, Y. Liu, F.S. Palumbo, Y. Luo and G.D. Prestwich, Biomaterials, 25, 1339 (2004); https://doi.org/10.1016/j.biomaterials.2003.08.014.

13. T. Ito, Y. Yeo, C. Highley, E. Bellas and D. Kohane, Biomaterials, 28, 3418 (2007);

https://doi.org/10.1016/j.biomaterials.2007.04.017. 
14. E.M. Milczek, Chem. Rev., 118, 119 (2018); https://doi.org/10.1021/acs.chemrev.6b00832.

15. R. Jin, L.S.M. Teixeira, P.J. Dijkstra, Z. Zhong, C.A. van Blitterswijk, M. Karperien and J. Feijen, Tissue Eng. Part A, 16, 2429 (2010); https://doi.org/10.1089/ten.tea.2009.0764.

16. J.W. Bae, J.H. Choi, Y. Lee and K.D. Park, J. Tissue Eng. Regen. Med., 9, 1225 (2015); https://doi.org/10.1002/term.1917.

17. L.S.M. Teixeira, S. Bijl, V.V. Pully, C. Otto, R. Jin, J. Feijen, C.A. van Blitterswijk, P.J. Dijkstra and M. Karperien, Biomaterials, 33, 3164 (2012); https://doi.org/10.1016/j.biomaterials.2012.01.001.

18. R. Jin, L.S.M. Teixeira, P.J. Dijkstra and M. Karperien, J. Biomater, 30, 2544 (2009);

https://doi.org/10.1016/j.biomaterials.2009.01.020.

19. F. Chen, S. Yu, B. Liu, Y. Ni, C. Yu, Y. Su, X. Zhu, X. Yu, Y. Zhou and D. Yan, Sci. Rep., 6, 20014 (2016); https://doi.org/10.1038/srep20014.

20. R. Jin, L.S.M. Teixeira, P.J. Dijkstra, C.A. van Blitterswijk, M. Karperien and J. Feijen, J. Control. Rel., 152, 186 (2011); https://doi.org/10.1016/j.jconrel.2011.01.031.

21. G. Tozzi, A. De Mori, A. Oliveira and M. Roldo, Materials, 9, 267 (2016); https://doi.org/10.3390/ma9040267.

22. Z.H. Derakhshan, B. Shaghaghi, M.P. Asl, M. Majidi, L. Ghazizadeh, A. Chegini and S. Bonakdar, Int. J. Polym. Mater. Polym. Biomater., 64, 919 (2015); https://doi.org/10.1080/00914037.2015.1030662.

23. E. Sanmartín-Masiá, S. Poveda-Reyes and G.G. Ferrer, Int. J. Polym. Mater. Polym. Biomater, 66, 280 (2017); https://doi.org/10.1080/00914037.2016.1201828.

24. J.A. Killion, L.M. Geever, D.M. Devine, C.L. Higginbotham and H. Farrell, Int. J. Polym. Mater. Polym. Biomater, 63, 641 (2014); https://doi.org/10.1080/00914037.2013.854238.

25. J.A. Killion, S. Kehoec, L.M. Geever, D.M. Devine, E. Sheehan, D. Boyd and C.L.Higginbotham, Mater. Sci. Eng. C, 33, 4203 (2013); https://doi.org/10.1016/j.msec.2013.06.013.

26. C.D.F. Moreira, S.M. Carvalho, H.S. Mansur and M.M. Pereira, Mater. Sci. Eng. C, 58, 1207 (2016); https://doi.org/10.1016/j.msec.2015.09.075.

27. T.P. Nguyen, B.H. Phuong Doan, D.V. Dang, C.K. Nguyen and N.Q. Tran, Adv. Nat. Sci.: NanoSci. Nanotechnol., 5, 015012 (2014); https://doi.org/10.1088/2043-6262/5/1/015012.

28. T.P. Nguyen and N.Q. Tran, Adv. Res., 7, 1 (2016); https://doi.org/10.9734/AIR/2016/25891.

29. B. Huang, M. Liu, Z. Long, Y. Shen and C. Zhou, Mater. Sci. Eng. C, 70, 303 (2017); https://doi.org/10.1016/j.msec.2016.09.001.
30. I.Y. Kim, S.J. Seo, H.S. Moon, M.K. Yoo, I.Y. Park, B.C. Kim and C.S. Cho, Biotechnol. Adv, 26, 1 (2008); https://doi.org/10.1016/j.biotechadv.2007.07.009.

31. N.Q. Tran, Y.K. Joung, E. Lih, K.M. Park and K.D. Park, Biomacromol., 11, 617 (2010); https://doi.org/10.1021/bm100047y.

32. N.Q. Tran, Y.K. Joung, E. Lih, K.M. Park and K.D. Park, Biomacromol., 12, 2872 (2011); https://doi.org/10.1021/bm200326g.

33. S. Sakai, K. Hirose, K. Taguchi, Y. Ogushi and K. Kawakami, Biomaterials, 30, 3371 (2009); https://doi.org/10.1016/j.biomaterials.2009.03.030.

34. X. Liu, L.A. Smith, J. Hu and P.X. Ma, Biomaterials, 30, 2252 (2009); https://doi.org/10.1016/j.biomaterials.2008.12.068.

35. K.M. Park, K.S. Ko, Y.K. Joung, H. Shin and K.D. Park, J. Mater. Chem., 21, 13180 (2011); https://doi.org/10.1039/c1jm12527c.

36. Y. Lee, J.W. Bae, D.H. Oh, K.M. Park, Y.W. Chun, H.J. Sung and K.D. Park, J. Mater. Chem. B, 1, 2407 (2013); https://doi.org/10.1039/C3TB00578J.

37. D.L. Nihouannen, A. Saffarzadeh, E. Aguado, E. Goyenvalle, O. Gauthier, F. Moreau, P. Pilet, R. Spaethe, G. Daculsi and P. Layrolle, J. Mater. Sci. Mater. Med., 18, 225 (2007); https://doi.org/10.1007/s10856-006-0684-7.

38. E.C. Victoria and F.D. Gnanam, Trends Biomater. Artif. Organs, 16, 12 (2002).

39. L. Nie, J. Suo, P. Zou and S. Feng, J. Nanomater., 2012, Article ID 213549 (2012); https://doi.org/10.1155/2012/213549.

40. M. Dziadek, E. Stodolak-Zych and K. Cholewa-Kowalska, Mater. Sci. Eng. C, 71, 1175 (2017); https://doi.org/10.1016/j.msec.2016.10.014.

41. T.T. Nguyen, T.P. Nguyen, T.T. Bui, T.T. Nguyen, H.B.S.L. Huynh, Q.S. Tran, T.P. Nguyen, C.K. Nguyen, D.H. Nguyen and N.Q. Tran, Vietnam J. Sci. Technol., 55(1B), 185 (2017); https://doi.org/10.15625/2525-2518/55/1B/12107.

42. D.Q. Wu, Y.X. Sun, X.D. Xu, S.X. Cheng, X.Z. Zhang and R.X. Zhuo, Biomacromolecules, 9, 1155 (2008); https://doi.org/10.1021/bm7010328.

43. J.J. Robinson, J. Cell. Biochem., 80, 139 (2001); https://doi.org/10.1002/1097-4644(20010101)80:1<139::AIDJCB130>3.0.CO;2-A.

44. Y.S. Wu, Y.H. Lee and H.C. Chang, Mater. Sci. Eng. C, 29, 237 (2009); https://doi.org/10.1016/j.msec.2008.06.018.

45. A. Bigi, E. Boanini, S. Panzavolta, N. Roveri and K. Rubini, J. Biomed. Mater. Res., 59, 709 (2002); https://doi.org/10.1002/jbm.10045. 